\title{
Lattice QCD calculations of transverse momentum-dependent parton distributions (TMDs)
}

\author{
M. Engelhardt1,a , B. Musch², T. Bhattacharya ${ }^{3}$, J. R. Green ${ }^{4}$, R. Gupta ${ }^{3}$, P. Hägler², S. Krieg ${ }^{5,6}$, \\ J. Negele ${ }^{7}$, A. Pochinsky ${ }^{7}$, A. Schäfer ${ }^{2}$, S. Syritsyn ${ }^{8}$, and B. Yoon ${ }^{3}$ \\ ${ }^{1}$ Department of Physics, New Mexico State University, Las Cruces, NM 88003, USA \\ ${ }^{2}$ Institut für Theoretische Physik, Universität Regensburg, 93040 Regensburg, Germany \\ ${ }^{3}$ Theoretical Division, Los Alamos National Laboratory, Los Alamos, NM 87545, USA \\ ${ }^{4}$ Institut für Kernphysik, Johannes Gutenberg-Universität Mainz, 55099 Mainz, Germany \\ ${ }^{5}$ Bergische Universität Wuppertal, 42119 Wuppertal, Germany \\ ${ }^{6}$ IAS, Jülich Supercomputing Centre, Forschungszentrum Jülich, 52425 Jülich, Germany \\ ${ }^{7}$ Center for Theoretical Physics, Massachusetts Institute of Technology, Cambridge, MA 02139, USA \\ ${ }^{8}$ Theory Center, Thomas Jefferson National Accelerator Facility, Newport News, VA 23606, USA
}

\begin{abstract}
An ongoing program of evaluating TMD observables within Lattice QCD is reviewed, summarizing recent progress with respect to several challenges faced by such calculations. These lattice calculations are based on a definition of TMDs through hadronic matrix elements of quark bilocal operators containing staple-shaped gauge connections. A parametrization of the matrix elements in terms of invariant amplitudes serves to cast them in the Lorentz frame preferred for a lattice calculation. Data on the naively T-odd Sivers and Boer-Mulders effects as well as the transversity TMD are presented.
\end{abstract}

\section{Introduction}

In the description of hadron structure, transverse momentum-dependent parton distribution functions [1] (TMDs) play a role complementary to generalized parton distributions (GPDs). Whereas GPDs encode information about the transverse spatial distribution of partons (through Fourier transformation with respect to the momentum transfer), TMDs contain information about the transverse momentum distribution of partons. Cast in a Lorentz frame in which the hadron of mass $m_{h}$ propagates with a large momentum in the 3-direction, $P^{+} \equiv\left(P^{0}+P^{3}\right) / \sqrt{2} \gg m_{h}$, the quark momentum components scale such that TMDs are principally functions $f\left(x, k_{T}\right)$ of the quark longitudinal momentum fraction $x=k^{+} / P^{+}$and the quark transverse momentum vector $k_{T}$, with the dependence on the component $k^{-} \equiv\left(k^{0}-k^{3}\right) / \sqrt{2} \ll m_{h}$ becoming ignorable in this limit. The function $f\left(x, k_{T}\right)$ will thus be regarded as having been integrated over $k^{-}$.

Experimentally, TMDs manifest themselves in angular asymmetries observed in processes such as semi-inclusive deep inelastic scattering (SIDIS) and the Drell-Yan (DY) process. Corresponding signatures have emerged at COMPASS, HERMES and JLab [2-4], and that has motivated targeting a significant part of the physics program at future experiments in this direction, e.g., at the upgraded

\footnotetext{
a e-mail: engel@nmsu.edu
} 
JLab $12 \mathrm{GeV}$ facility and at the proposed electron-ion collider (EIC). Relating the experimental signature to the hadron structure encoded in TMDs requires a suitable factorization framework, the one having been advanced in [5-8] being particularly well-suited for connecting phenomenology to Lattice QCD. Factorization in the TMD context is considerably more involved than standard collinear factorization, with the resulting TMDs in general being process-dependent, via initial and/or final state interactions between the struck quark and the hadron remnant.

\section{Definition of TMD observables}

The definition of TMD observables amenable to lattice evaluation has been laid out in detail in [9]. Summarizing briefly, the starting point is the fundamental correlator

$$
\widetilde{\Phi}_{\text {unsubtr. }}^{[\Gamma]}(b, P, S, \ldots) \equiv \frac{1}{2}\langle P, S|\bar{q}(0) \Gamma \mathcal{U}[0, \eta v, \eta v+b, b] q(b)| P, S\rangle
$$

where $S$ denotes the spin of the hadron and $\Gamma$ stands for an arbitrary $\gamma$-matrix structure. The stapleshaped gauge connection $\mathcal{U}$ follows straight-line paths connecting the positions given in its argument; the unit vector $v$ thus specifies the direction of the staple, whereas $\eta$ parametrizes its length. The presence of $\mathcal{U}$ introduces divergences in $\widetilde{\Phi}_{\text {unsubtr. }}^{[\Gamma]}$ additional to the wave function renormalizations of the quark operators; these divergences accordingly must ultimately be compensated by additional "soft factors", which are expected to be multiplicative and do not need to be specified in detail here, since only appropriate ratios in which they then presumably cancel will ultimately be considered. In order to regularize rapidity divergences, the staple direction $v$ is taken slightly off the light cone into the space-like region $[5,6]$, with perturbative evolution equations governing the approach to the light cone [7]. A useful parameter characterizing how close $v$ is to the light cone is the Collins-Soper evolution parameter $\hat{\zeta}=v \cdot P /(|v||P|)$, in terms of which the light cone is approached for $\hat{\zeta} \rightarrow \infty$.

The correlator (1) can be decomposed in terms of invariant amplitudes $\widetilde{A}_{i B}$. The components relevant for the Sivers and Boer-Mulders effects, as well as the transversity, discussed below, are

$$
\begin{aligned}
\frac{1}{2 P^{+}} \widetilde{\Phi}_{\text {unsubtr. }}^{\left[\gamma^{+}\right]} & =\widetilde{A}_{2 B}+i m_{h} \epsilon_{i j} b_{i} S_{j} \widetilde{A}_{12 B} \\
\frac{1}{2 P^{+}} \widetilde{\Phi}_{\text {unsubtr. }}^{\left[i \sigma^{i+} \gamma^{5}\right]} & =i m_{h} \epsilon_{i j} b_{j} \widetilde{A}_{4 B}-S_{i} \widetilde{A}_{9 B}-i m_{h} \Lambda b_{i} \widetilde{A}_{10 B}+m_{h}\left[(b \cdot P) \Lambda-m_{h}\left(b_{T} \cdot S_{T}\right)\right] b_{i} \widetilde{A}_{11 B}
\end{aligned}
$$

where $\Lambda$ denotes the hadron helicity (i.e., $S^{+}=\Lambda P^{+} / m_{h}, S^{-}=-\Lambda m_{h} / 2 P^{+}$). These amplitudes are useful in that they can be evaluated in any desired Lorentz frame, including a frame that is particularly suited for the lattice calculation. Specializing, for present purposes, to TMDs integrated over momentum fraction $x$, by considering specifically ${ }^{1} b \cdot P=0$, they serve to define the "generalized Sivers shift"

$$
\left\langle k_{y}\right\rangle_{T U}\left(b_{T}^{2}, \ldots\right)=-m_{h} \widetilde{A}_{12 B}\left(-b_{T}^{2}, \ldots\right) / \widetilde{A}_{2 B}\left(-b_{T}^{2}, \ldots\right)=m_{h} \tilde{f}_{1 T}^{\perp[1](1)}\left(b_{T}^{2}, \ldots\right) / \tilde{f}_{1}^{[1](0)}\left(b_{T}^{2}, \ldots\right)
$$

where the right-hand expression introduces the notation in terms of Fourier-transformed TMD moments, for details, cf. [9, 10]; as discussed in [11], the use of Fourier-transformed TMDs is also advantageous in the analysis of experimental data. In the $b_{T} \rightarrow 0$ limit, (4) formally represents the average transverse momentum $k_{y}$ of unpolarized (" $U$ ") quarks orthogonal to the transverse (" $T$ ") spin of the hadron, normalized to the corresponding number of valence quarks. Analogously, one can also extract the generalized Boer-Mulders shift

$$
\left\langle k_{y}\right\rangle_{U T}\left(b_{T}^{2}, \ldots\right)=m_{h} \widetilde{A}_{4 B}\left(-b_{T}^{2}, \ldots\right) / \widetilde{A}_{2 B}\left(-b_{T}^{2}, \ldots\right)=m_{h} \tilde{h}_{1}^{\perp[1](1)}\left(b_{T}^{2}, \ldots\right) / \tilde{f}_{1}^{[1](0)}\left(b_{T}^{2}, \ldots\right)
$$

\footnotetext{
${ }^{1}$ The treatment can be readily expanded to nonzero $b \cdot P$, providing access to the $x$-dependence of TMDs.
} 
which, in the $b_{T} \rightarrow 0$ limit, formally represents the average transverse momentum $k_{y}$ of quarks polarized in the transverse (" $T$ ") direction orthogonal to the $k_{y}$-direction in an unpolarized (" $U$ ") hadron, normalized to the corresponding number of valence quarks. Similarly, one can introduce a generalized tensor charge via the ratio of Fourier-transformed moments of the transversity and unpolarized TMDs,

$$
\tilde{h}_{1}^{[1](0)}\left(b_{T}^{2}, \ldots\right) / \tilde{f}_{1}^{[1](0)}\left(b_{T}^{2}, \ldots\right)=-\left[\widetilde{A}_{9 B}\left(-b_{T}^{2}, \ldots\right)-m_{h}^{2} b^{2} \widetilde{A}_{11 B}\left(-b_{T}^{2}, \ldots\right) / 2\right] / \widetilde{A}_{2 B}\left(-b_{T}^{2}, \ldots\right) .
$$

In the $b_{T} \rightarrow 0$ limit, this formally reduces to the tensor charge, normalized to the corresponding number of valence quarks. The ratios (4)-(6) are designed to cancel both multiplicative soft factors associated with the gauge connection $\mathcal{U}$ as well as wave function renormalizations attached to the quark operators in (1) at finite physical separation $b$. It should be emphasized that the construction presented up to this point has been developed within continuum QCD; transferring it verbatim to Lattice QCD constitutes a working assumption which ultimately requires further consideration. In particular, the breaking of space-time symmetries engendered by the lattice discretization may imply changes to the purely multiplicative nature of the soft factors and renormalization constants, and thus invalidate the cancellations invoked in the construction of the ratios (4)-(6). Pending a foundational investigation of these issues within the lattice formulation, empirical insight into possible renormalization effects can be obtained by studying the stability of TMD ratios such as (4)-(6) under substantial changes of the lattice discretization. This constitutes one of the topics addressed in the presentation of numerical results below.

\section{Lattice evaluation and results}

To access observables such as (4)-(6) within Lattice QCD, one calculates hadron matrix elements of the type (1) and then decomposes them into invariant amplitudes, as given in (2) and (3). For this to be possible, it is crucial to work in a scheme where the four-vectors $b$ and $v$ are generically space-like, for the following reason: By employing a Euclidean time coordinate to project out hadron ground states via Euclidean time evolution, Lattice QCD cannot straightforwardly accomodate operators containing Minkowski time separations. The operator of which one takes matrix elements thus has to be defined at a single time. Only if both $b$ and $v$ are space-like is there no obstacle to boosting the problem to a Lorentz frame in which $b$ and $v$ are purely spatial, and evaluating $\widetilde{\Phi}_{\text {unsubtr. }}^{[\Gamma]}$ in that frame. The results extracted for the invariant amplitudes $\widetilde{A}_{i B}$ are then immediately valid also in the original frame in which (1) was initially defined, thus completing the determination of quantities of the type (4)-(6).

Since, in a numerical lattice calculation, the staple extent $\eta$ necessarily remains finite, two extrapolations must be performed from the generated data, namely, the one to infinite staple length, $\eta \rightarrow \infty$, and the extrapolation of the staple direction towards the light cone, $\hat{\zeta} \rightarrow \infty$. As discussed below, the former extrapolation is fairly straightforward. On the other hand, the extrapolation $\hat{\zeta} \rightarrow \infty$ constitutes a considerable challenge. Given that $v$ is purely spatial in the Lorentz frame used for the lattice calculation, the accessible range of $\hat{\zeta}$ is determined by the available hadron spatial momenta $P$ (in units of the hadron mass). The numerical signal achieved in lattice calculations rapidly deteriorates with rising hadron momentum, and thus only a very limited set of $\hat{\zeta}$ can be accessed. Data from a dedicated study of the Boer-Mulders shift in a pion [12] are discussed below to address this challenge; the lower mass of the pion compared to the nucleon facilitates reaching higher $\hat{\zeta}$ and makes a controlled extrapolation possible.

Finally, a general challenge faced by Lattice QCD calculations is reaching the physical pion mass. The computational expense of treating quark masses corresponding to the physical point has hitherto precluded the generation of lattice TMD data at that point. Instead, data are produced at artificially 

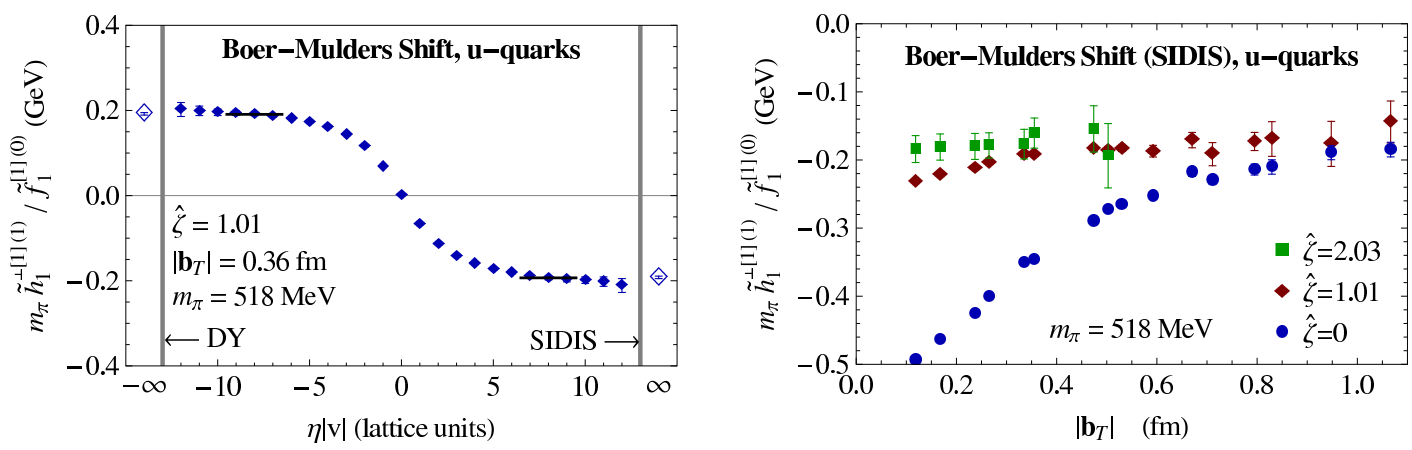

Figure 1. Generalized Boer-Mulders shift in a pion as a function of staple extent at fixed quark separation $b_{T}$ and Collins-Soper evolution parameter $\hat{\zeta}$ (left); and as a function of $b_{T}$ in the $\eta \rightarrow \infty$ SIDIS limit, at three different values of $\hat{\zeta}$ (right).
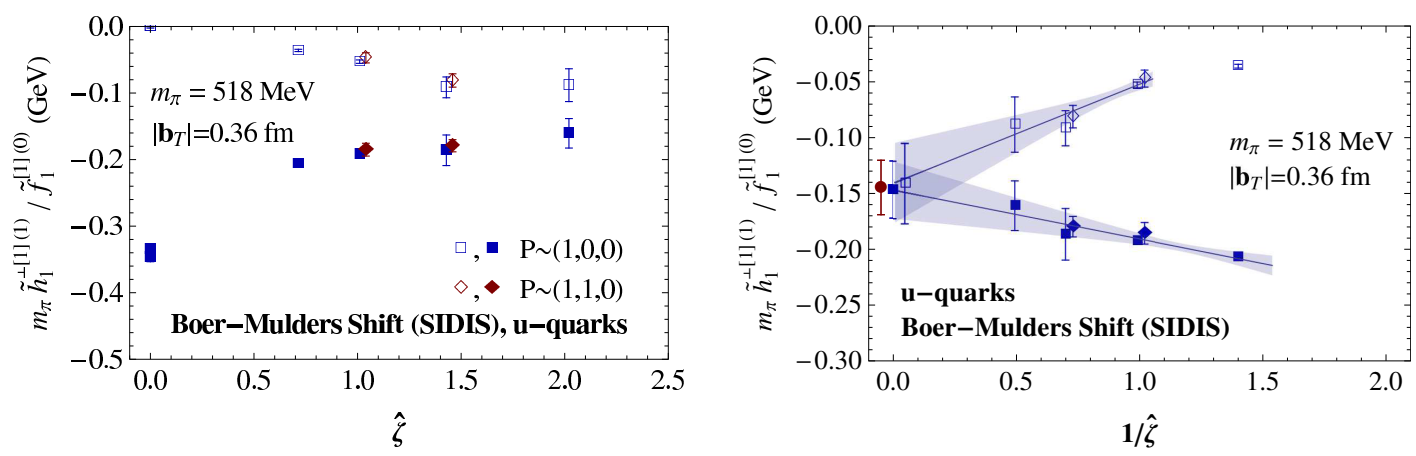

Figure 2. Generalized Boer-Mulders shift in a pion in the SIDIS limit as a function of Collins-Soper evolution parameter $\hat{\zeta}$ at fixed $b_{T}$ (left); and as a function of $1 / \hat{\zeta}$ together with fits using the form $c+d / \hat{\zeta}$ (right). Shown are both the full shift (filled symbols) as well as a partial contribution (open symbols), cf. comments in the main text. Fit curves are for separate fits to the two cases; the extrapolated data point shown with a circle symbol results from a simultaneous fit to both.

high pion masses. To also obtain preliminary insight into the pion mass dependence of TMD observables, data at differing pion masses are juxtaposed below.

Focusing first on the issue of $\hat{\zeta}$-extrapolation, Figs. 1 and 2 display data for the generalized BoerMulders shift (5) in a pion [12], obtained in a mixed action scheme employing domain wall valence quarks on a MILC 2+1-flavor asqtad quark ensemble with a lattice spacing of $a=0.12 \mathrm{fm}$ and a pion mass of $m_{\pi}=518 \mathrm{MeV}$. In the pion case, the isovector quark combination vanishes; the shown data are for $u$-quarks alone, with the corresponding disconnected contributions omitted. Fig. 1 (left) exhibits results obtained at a given quark separation $b_{T}$ and a given staple direction characterized by $\hat{\zeta}$, as a function of the staple extent. The T-odd behavior of the observable is evident, with $\eta \rightarrow \infty$ corresponding to the SIDIS limit, whereas $\eta \rightarrow-\infty$ yields the DY limit. The data level off to approach clearly identifiable plateaux as the staple length grows. The limiting SIDIS and DY values, represented by the open symbols, are extracted by imposing antisymmetry in $\eta$, allowing one to appropriately average the $\eta \rightarrow \pm \infty$ plateau values. Fig. 1 (right) summarizes the SIDIS limit values as a function of $b_{T}$ for three different $\hat{\zeta}$. Remarkably, the $b_{T}$-dependence of the Boer-Mulders shift flattens as $\hat{\zeta}$ is increased, and the data for different $\hat{\zeta}$ approach each other at large $b_{T}$. It would be useful to understand 

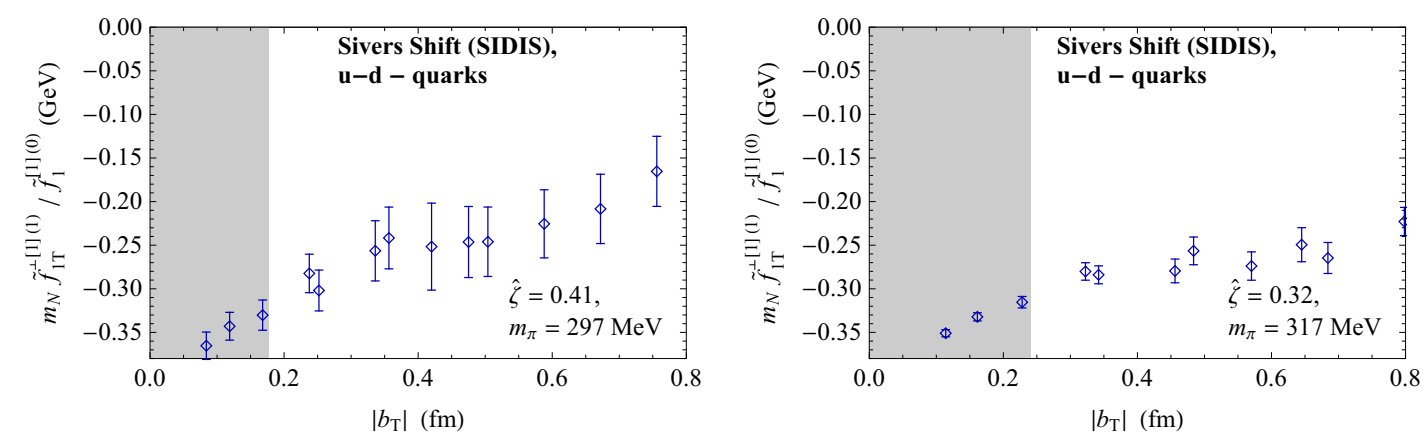

Figure 3. Generalized Sivers shift in a nucleon as a function of $b_{T}$ in the $\eta \rightarrow \infty$ SIDIS limit, at a fixed $\hat{\zeta}$, in a fine lattice domain wall fermion calculation (left) and a coarse lattice clover fermion calculation (right), cf. main text for details.

this behavior in detail. Fig. 2 focuses on a particular value of $b_{T}$, displaying the $\hat{\zeta}$-dependence of both the full Boer-Mulders shift as well as a certain partial contribution which vanishes at $\hat{\zeta}=0$, but dominates the quantity at large $\hat{\zeta}$; comparison of the full Boer-Mulders shift with the partial contribution thus can give an indication of convergence towards the large $\hat{\zeta}$ limit. For further details, cf. $[9,12]$. The partial contribution already furnishes roughly one half of the full shift at the highest $\hat{\zeta}$ reached, signaling that the calculation has covered a significant part of the evolution to large $\hat{\zeta}$. The right-hand panel of Fig. 2 shows an extrapolation to the large $\hat{\zeta}$ limit using the functional form $c+d / \hat{\zeta}$ (analogous fits using the form $c+d / \hat{\zeta}^{2}$ are seen to be inferior [12]). Given that the range of $\hat{\zeta}$ accessed numerically does not clearly overlap with the regime in which perturbative evolution equations become applicable, this form should be viewed as no more than a physically motivated ad hoc ansatz. The fits to the full and partial data converge to compatible values, agreeing also with a combined fit to both data sets. This buttresses confidence in the extrapolations and demonstrates that lattice calculations can achieve a signal for the Boer-Mulders shift of sufficient quality such that it survives taking the $\hat{\zeta} \rightarrow \infty$ limit.

Turning to the issue of the dependence of TMD ratios on the lattice discretization, which provides an empirical test of whether renormalization effects indeed cancel in such observables, Fig. 3 shows representative results for the generalized Sivers shift (4) in the nucleon. Results for the isovector, $u-d$ quark combination are displayed; in this channel, couplings of the operator insertion to disconnected quark loops in the nucleon, which have not been evaluated, cancel. The figures juxtapose results obtained on a RBC/UKQCD 2+1-flavor domain wall fermion ensemble featuring a lattice spacing of $a=0.084 \mathrm{fm}$ and a pion mass of $m_{\pi}=297 \mathrm{MeV}$ with results obtained on a 2+1-flavor clover fermion ensemble provided by K. Orginos and collaborators in the Jefferson Lab lattice group; the latter has a lattice spacing of $a=0.114 \mathrm{fm}$ and a pion mass of $m_{\pi}=317 \mathrm{MeV}$. The two ensembles thus have very similar pion masses, but differ substantially in the lattice discretization. The domain wall fermion ensemble not only features a considerably finer spacing, but also respects chiral symmetry to the largest extent possible, whereas the clover fermion ensemble strongly breaks the continuum symmetry. Fig. 3, analogously to Fig. 1 (right), shows the SIDIS limit results as a function of $b_{T}$ at a given $\hat{\zeta}$, where the shaded area below $\left|b_{T}\right|=2 a$ indicates the region where the data may be significantly affected by finite lattice cutoff effects. The results for the Sivers shift obtained on the two ensembles in question are compatible, despite the considerable differences in terms of discretization scheme. This suggests that the renormalization effects embodied in soft factors and wave function renormalization constants indeed cancel in TMD ratios also in the lattice formulation. 

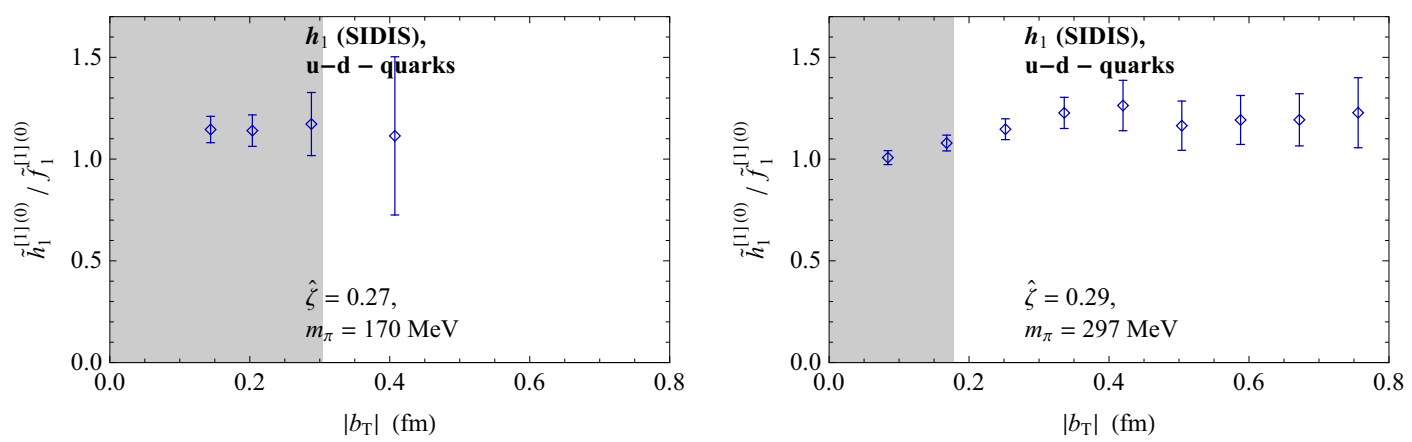

Figure 4. Generalized tensor charge as a function of $b_{T}$ in the $\eta \rightarrow \infty$ SIDIS limit, at a fixed $\hat{\zeta}$, in domain wall fermion calculations at $m_{\pi}=170 \mathrm{MeV}$ (left) and $m_{\pi}=297 \mathrm{MeV}$ (right).

Finally, a preliminary indication of the pion mass dependence of TMD ratios in the chiral regime is given by the juxtaposition in Fig. 4. It compares data for the generalized tensor charge of the nucleon (6) obtained on two RBC/UKQCD 2+1-flavor domain wall fermion ensembles, one with a lattice spacing of $a=0.144 \mathrm{fm}$ and a pion mass of $m_{\pi}=170 \mathrm{MeV}$, and the other with a lattice spacing of $a=0.084 \mathrm{fm}$ and a pion mass of $m_{\pi}=297 \mathrm{MeV}$, cf. also Fig. 3 (left). The lighter pion mass data are affected by considerably stronger statistical fluctuations; the $m_{\pi}=170 \mathrm{MeV}$ calculation employed 8 source-sink pairs on each of 310 lattices, i.e., 2480 samples, for each matrix element; the $m_{\pi}=297 \mathrm{MeV}$ calculation 8 source-sink pairs on 533 lattices, i.e., 4264 samples. Fig. 4 displays, analogously to Fig. 3, the SIDIS limit data for the generalized tensor charge as a function of $b_{T}$ at a given $\hat{\zeta}$. Keeping in mind that the sizeable statistical uncertainties at $m_{\pi}=170 \mathrm{MeV}$ preclude a stringent comparison, the results at the two pion masses are compatible; no significant variation with $m_{\pi}$ is apparent even this far into the chiral regime.

\section{Summary and outlook}

TMDs can be formally defined in terms of hadronic matrix elements of quark bilocal operators containing staple-shaped gauge connections, which incorporate final/initial state effects in SIDIS/DY processes. Evaluating such matrix elements within Lattice QCD, one encounters several challenges. For one, to cancel multiplicative soft factors and renormalization constants, appropriate ratios of Fouriertransformed TMDs, cf. (4)-(6), are constructed. However, the breaking of space-time symmetries engendered by the lattice discretization may imply changes to the purely multiplicative nature of the soft factors and renormalization constants contained in the continuum definition; this possibility was addressed empirically by studying the universality of TMD ratios under changes of the discretization scheme. Secondly, the gauge connection staples are generically taken off the light cone to regularize rapidity divergences, with the Collins-Soper parameter $\hat{\zeta}$ controlling the approach to the light cone. A dedicated study of pion TMD ratios demonstrated that the attendant extrapolation in $\hat{\zeta}$ is feasible within Lattice QCD calculations. Thirdly, TMD calculations must ultimately approach the physical pion mass; the pion mass dependence of TMD ratios has been explored, and no significant variations with pion mass have hitherto been observed, although more accurate, higher statistics data at light pion masses are needed to draw any substantive conclusions about the $m_{\pi}$-dependence of TMD ratios as the physical pion mass is approached. These studies buttress the ongoing program of calculating TMD observables within Lattice QCD presented here. Beyond the TMD observables discussed above, the developed methods are also being put to use to study generalized observables containing a nonzero 
momentum transfer in (1). These can be related to quark orbital angular momentum in the nucleon, since they provide mixed quark position and momentum information in the transverse plane. First exploratory data in this direction are becoming available.

\section{Acknowledgements}

Computations were performed using resources provided by the U.S. DOE Office of Science through the National Energy Research Scientific Computing Center (NERSC), a DOE Office of Science User Facility, under Contract No. DE-AC02-05CH11231, as well as through facilities of the USQCD Collaboration, employing the Chroma software suite [13]. The MILC and RBC/UKQCD collaborations are gratefully acknowledged for providing gauge ensembles analyzed in this work, as are K. Orginos (supported by DOE grant DE-FG02-04ER41302) and the Jefferson Lab lattice group (supported by DOE grant DE-AC05-06OR23177, under which Jefferson Science Associates, LLC, operates Jefferson Laboratory). Support by the Heisenberg-Fellowship program of the DFG (P.H.), SFB/TRR-55 (S.K.,A.S.), the PRISMA Cluster of Excellence at the University of Mainz (J.G.), and the RIKEN Foreign Postdoctoral Researcher Program (BNL) as well as the Nathan Isgur Fellowship (JLab) (S.S.) is acknowledged. This work was furthermore supported by the U.S. DOE and the Office of Nuclear Physics through grants DE-FG02-96ER40965 (M.E.), DE-SC0011090 (J.N.) and DE-FC0206ER41444 (A.P.). R.G., T.B. and B.Y. are supported by DOE grant DE-KA-1401020 and the LDRD program at LANL.

\section{References}

[1] D. Boer, M. Diehl, R. Milner, R. Venugopalan, W. Vogelsang, et al., arXiv: 1108. 1713.

[2] M. Alekseev, et al., COMPASS Collaboration, Phys. Lett. B673, 127 (2009).

[3] A. Airapetian, et al., HERMES Collaboration, Phys. Rev. Lett. 103, 152002 (2009).

[4] H. Avakian, et al., CLAS Collaboration, Phys. Rev. Lett. 105, 262002 (2010).

[5] S. M. Aybat and T. C. Rogers, Phys. Rev. D 83, 114042 (2011).

[6] J. C. Collins, Foundations of Perturbative QCD (Cambridge University Press, Cambridge, 2011).

[7] S. M. Aybat, J. C. Collins, J.-W. Qiu and T. C. Rogers, Phys. Rev. D 85, 034043 (2012).

[8] J. C. Collins and T. C. Rogers, Phys. Rev. D 87, 034018 (2013).

[9] B. Musch, P. Hägler, M. Engelhardt, J. W. Negele and A. Schäfer, Phys. Rev. D 85, 094510 (2012).

[10] D. Boer, L. Gamberg, B. Musch and A. Prokudin, JHEP 1110, 021 (2011).

[11] M. Aghasyan, et al., JHEP 1503, 039 (2015).

[12] M. Engelhardt, P. Hägler, B. Musch, J. W. Negele and A. Schäfer, arXiv: 1506.07826.

[13] R. G. Edwards and B. Joó, SciDAC Collaboration, Nucl. Phys. Proc. Suppl. 140, 832 (2005). 